\title{
Gamificação personalizada: um estudo de mapeamento sistemático
}

\author{
Aline A. Morais' ${ }^{1}$ Lafayette B. Melo' \\ Unidade Acadêmica de Informática - Instituto Federal da Paraíba (IFPB) \\ Caixa Postal 58015-435 - João Pessoa - PB - Brasil \\ \{araujo,morais\}@academico.ifpb.edu.br, laayette.melodifpb.edu.br
}

\begin{abstract}
This paper aims to investigate the types of frameworks and models of personalized gamification, their adaptations, and in which sectors it is present. The systematic mapping was carried out from December 2020 to April 2021 in the following search engines: ACM, IEEE Xplore, SciELO, SCOPUS, and Springer. A total of 2,604 studies were found in the period from 2016 to 2021; of these, 20 met the inclusion and exclusion criteria defined in the protocol. In total, 3 frameworks and 3 proposals were found. Thus, we were able to verify the frameworks used, those that are emerging, and the adaptations made, in addition to having an insight into the sectors that are using personalized gamification, especially education.
\end{abstract}

Resumo. Este trabalho tem objetivo investigar os tipos de framework $e$ modelos de gamificação personalizada, as suas adaptações e em quais setores está presente. O mapeamento sistemático foi realizado de dezembro de 2020 a abril de 2021, nos seguintes mecanismos de buscas: ACM, IEEE Xplore, SciELO, SCOPUS e Springer. Foram encontrados 2.604 estudos no período de 2016 a 2021; destes, 20 atenderam aos critérios de inclusão e exclusão definidos no protocolo. No total, foram encontrados 3 frameworks e 3 propostas. Assim, pudemos verificar os frameworks utilizados, os que estão surgindo e as adaptações realizadas, além de ter uma visão dos setores que estão utilizando a gamificação personalizada, especialmente a educação.

\section{Introdução}

A motivação para descobrir formas mais eficazes de proporcionar uma aprendizagem prazerosa e motivadora cresceu ao longo dos anos. Corriqueiramente se faz necessário descobrir as características da turma a partir dos alunos, e assim aplicar algum tipo de processo de ensino. Porém, diversas das aplicações baseadas em gamificação pecam nesta função, já que são implementadas com pedaços da mecânica, dinâmica e componentes de jogos e não estabelecem um design conciso e.g. [Mora et al. 2017].

Desta forma, ao dar início à construção de uma gamificação é fundamental entender os métodos e estruturas que existem e se encaixam em alguma problemática, para posteriormente construir algo com base nos objetivos específicos de cada gamificação e.g. [Mora et al. 2017]. Na literatura se discute sobre um bom design gamificado, que seria a junção de elementos de jogos, e os objetivos a serem almejados, para, com isso, minimizar resultados negativos que a gamificação pode acarretar, como 
indiferença, comportamentos indesejados ou a falta de motivação e engajamento e.g. [Toda; Valle e Isotani 2017].

Os frameworks vieram com o objetivo de ajudar no processo de design da gamificação e, desta forma, viabilizar os recursos fundamentais para a aplicação gamificada e assim se acautelar ao final da prática com engajamento efetivo. Os pesquisadores utilizam da gamificação com o intuito de engajar as pessoas, melhorar a experiência do usuário e torná-la agradável e alterar atividades que são desagradáveis e.g. [Hamari, Koivisto e Sarsa 2014], [Seaborn e Fels 2015].

Propiciar essa quebra de paradigma, de uma atividade que muda de desagradável para agradável, não é uma tarefa fácil já que aborda diversos fatores como pessoas, e também tecnologias, métodos, frameworks, mecânicas, dinâmicas e componentes para atraírem o interesse dos usuários. Considera-se também a condução de mapeamentos sistemáticos em tópicos de pesquisa nos quais poucas evidências estão disponíveis na literatura e.g. [Kitchenham e Charters 2007].

Esse estudo enfatizou a busca por trabalhos aplicados a gamificação personalizada e seus frameworks relacionados. A partir disso foram elencadas as questões de pesquisa, bem como as questões de inclusão e exclusão de artigos, fazendo o número de estudos reduzir consideravelmente e focando somente no que é importante para o mapeamento.

\section{Fundamentação Teórica}

Já faz alguns anos que a expressão gamificação é um termo bem utilizado na área industrial e no marketing, tendo como finalidade aumentar o engajamento de colaboradores até mesmo na fidelização dos clientes da empresa, mas especialmente em capacitações e treinamentos. A gamificação tem emergido e se estabilizado na área da educação, com o crescente número de publicações acadêmicas sobre o tema e.g. [Lamprinou e Paraskeva 2015]. Mas sua utilização pedagógica de forma lúdica pelos educadores, se dá algumas vezes com o uso de tecnologias digitais. A seguir, estão descritas algumas pesquisas bibliográficas, detalhando mais os assuntos supracitados.

O mapeamento sistemático de e.g. Souza et al. (2017) teve foco no contexto da educação em Engenharia de Software. Em seus resultados analisados conclui-se que o maior número de estudos em gamificação estava ligado às disciplinas de computação.

Em Oliveira (2018) foi realizado um mapeamento sistemático de literatura para verificar as vantagens e desvantagens dos processos de gamificação, os recursos e ferramentas utilizadas no cotidiano escolar e foi feita uma reflexão das experiências gamificadas na educação básica.

Na pesquisa de e.g. Poffo e Augustini (2018), os pesquisadores verificaram a motivação dos alunos conforme foram implementados elementos variados na gamificação. O estudo explica que existem vários itens para avaliar: utilização do jogo, design do jogo para motivação, o interesse no jogo e o conteúdo aplicado.

No mapeamento de e.g. Andrade (2019), foi feito um direcionamento para identificar e categorizar as pesquisas disponíveis na literatura que utilizam gamificação no Moodle. Teve como base os seguintes aspectos: os elementos de jogos utilizados, a integração com o Moodle, objetivos e resultados obtidos da aplicação da gamificação, contexto da avaliação empírica, maturidade dos trabalhos e histórico da pesquisa. 
Pôde-se observar que, nas pesquisas anteriormente citadas, os mapeamentos tocam em vários assuntos de gamificação, mas não há relações com estudos sobre personalização e os respectivos frameworks utilizados. Nesse sentido, esta pesquisa tomou como base alguns procedimentos desses trabalhos, mas em busca de um mapeamento que tratasse de forma mais específica a personalização de ambientes com empregos de possíveis frameworks, no sentido se suprir como outras pesquisas podem se debruçar sobre esse assunto, compreendendo pontos de partida de uso, mesmo que seja para algumas adaptações. Na próxima seção, apresentamos como foi realizada toda a metodologia da pesquisa com seu procedimento passo a passo para se chegar aos dados apresentados posteriormente.

\section{Metodologia de Pesquisa}

Esse estudo é uma pesquisa exploratória que foi conduzida a partir de uma busca na literatura sobre a utilização de gamificação personalizada através do método de mapeamento sistemático. Esse método é planejado, estruturado e controlado para fornecer uma estrutura que outros pesquisadores possam replicá-los. O processo de mapeamento sistemático segundo e.g. Peterson, Vakkalanka e Kuzniarz (2015) realiza uma classificação e rastreia a contribuição que existe na literatura com o objetivo de proporcionar uma visão geral da área, assim colaborando na assimilação do estado da arte e identificação de possíveis melhorias ou lacunas. Este processo possui as cinco etapas apresentadas na Figura 1. Cada uma dessas etapas será detalhada posteriormente.

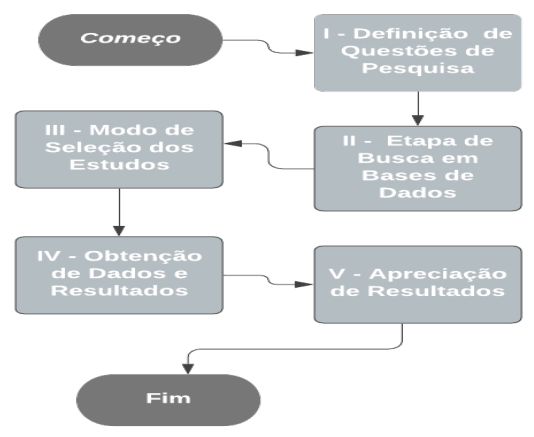

Figura 1. Detalhamento das cinco etapas no mapeamento sistemático. Figura adaptada a partir de Peterson, Vakkalanka e Kuzniarz [2015].

\subsection{Definição das questões de pesquisa}

Nesta etapa formulamos as perguntas para as questões de pesquisas (QP) baseadas nos objetivos desta pesquisa. E foram as seguintes:

QP1: Quais foram os modelos ou frameworks de gamificação personalizada utilizados na gamificação?

QP2: Dentre as pesquisas encontradas ocorreu algum tipo de adaptação do modelo ou framework utilizado?

QP3: Em quais setores foi utilizada a gamificação personalizada? 


\subsection{Etapa de busca nos mecanismos de busca}

Nessa etapa procura-se definir a estratégia de busca a ser feita durante a pesquisa. Foi planejada a string de busca adequada para a pesquisa. Guiamo-nos pelas seguintes sugestões de e.g. [Peterson, Vakkalanka e Kuzniarz 2015] e [Kitchenham e Charters 2007] a partir da finalidade de reduzir vieses:

I - Reconhecimento sobre conceitos importantes com base nas questões de pesquisa;

II - Pesquisar em títulos de artigos, revistas, anais e conferências relevantes;

III - Atentar a termos alternativos, sinônimos e abreviações para as palavras-chaves; e

IV - Utilizar operadores booleanos "OU" para agrupar os sinônimos e "E" para agrupar termos diferentes.

Foram identificadas palavras-chaves que se tornaram os argumentos para a busca, com base em 3 termos diferentes que são: gamificação, framework e personalização e seus diversos sinônimos e respectivos plurais.

Optamos por bases de dados científicas que fornecem ferramentas ou mecanismos para realizar buscas automáticas e online e possuem fontes relevantes na área de tecnologia. A partir disto selecionamos as seguintes bases de dados: ACM Digital Library, IEEE Xplore, SciELO, Scopus e Springer. Esta etapa aconteceu durante o período de 1 de dezembro de 2020 até 27 de abril de 2021 e foram encontrados 2604 estudos. A Tabela 1 apresenta os resultados encontrados em cada base de dados.

Tabela 1. Quantidade de estudos obtidos após busca em cada mecanismo de busca com base nos dados obtidos

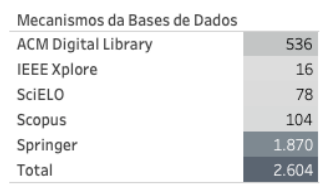

Fonte: Elaborada pelos autores com base nos estudos primários (2016 - 2021).

Essa etapa de busca nos retornou estudos a partir de 5 bases de dados, e através delas totalizaram 2604 estudos que seguiram para outra parte do mapeamento sistemático, que é o modo de seleção dos estudos.

\subsection{Modo de seleção de estudos}

Para delimitar de maneira mais adequada com relação ao propósito do mapeamento, deve-se utilizar uma delimitação com critérios de inclusão (CI) e critérios de exclusão (CE).

Critérios de Inclusão:

CI.1: Os estudos devem contemplar a utilização de modelo ou framework de gamificação personalizada;

CI.2: Estudos que apresentam textos completos (consideram-se trabalhos com, no mínimo, quatro páginas);

CI.3: O estudo deve estar na internet e com seu material totalmente disponível na web; e

CI.4: O estudo deve ser publicado de 2016 a 2021. 
Critérios de Exclusão:

CE.1: Estudos que não tenham na sua escrita o idioma português ou inglês;

CE.2: Estudos que tenham alguma forma de cobrança para o acesso; e

CE.3: Estudos duplicados - quando o mesmo estudo estiver disponível em diferentes fontes de busca, apenas a primeira pesquisa será considerada.

Os estudos obtidos na etapa anterior foram utilizados para servir de entrada nesta etapa. Os modos de seleções CI e o CE, demonstrados anteriormente, foram utilizados como base para obter os estudos relevantes. A lista de inclusão foi feita por ordem alfabética com base nos mecanismos de busca. Aplicamos os critérios mencionados acima para filtrar os estudos relevantes. A leitura dos estudos deu-se em 3 etapas, que foram:

1. Leitura de título, resumo e as palavras-chaves;

2. Leitura da Introdução e Conclusão; e

3. Leitura completa do estudo.

Essas três etapas de leitura foram realizadas para averiguar uma possível maneira de assegurar a relevância dos estudos. Com isso, após realizar todas as análises dos CI e CE e finalizar as etapas de leitura, foram considerados 20 estudos relevantes. A Tabela 2 traz todos os estudos retornados nos mecanismos de busca, com os resultados depois das três etapas de leitura, com a separação para cada base de dados científica e a quantidade final de estudos relevantes.

Tabela 2. Apresenta a quantidade total obtida em cada mecanismo de busca e os estudos relevantes de cada um destes.

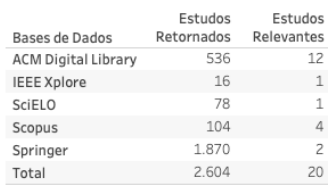

Fonte: Elaborada pelos autores com base nos estudos primários (2016 - 2021).

Apenas uma versão do documento foi arquivada e os duplicados eram removidos se indexados por mais de um mecanismo de busca. Como resultado final, obtivemos um total de 20 artigos relevantes. A ACM foi a base de dados que retornou a maior quantidade de estudos relevantes, já a IEEE e ScIELO trouxeram apenas um artigo relevante cada. Na ScIELO vários estudos estavam cobrando pelo acesso (CE2), aconteceu algo parecido nos estudos encontrados na Springer que não são gratuitos (CE2) ou não estão totalmente disponíveis na internet (CI3). Na Scopus identificamos alguns artigos que estavam duplicados (CE3). A seguir apresentaremos os dados obtidos a partir desse mapeamento sistemático.

\subsection{Modo de seleção dos estudos}

A partir dos estudos que atendem os critérios de aceitação e critérios de exclusão, obtivemos uma lista de estudos primários que serve como base para extração de dados fundamental para responder às questões de pesquisa $(\mathrm{QP})$. Após leitura dos documentos, obtemos informações sobre vários aspectos como: ano, local de publicação, mecanismo de busca de origem, autores e países. A tabela 3 apresenta a lista de artigos enumerados 
para melhor apresentação dos dados posteriormente. A Tabela 4 apresenta a relação entre a quantidade de estudos primários publicados de 2016 até 2021. Percebemos um crescente interesse no tema no ano de 2020. A Tabela 5 apresenta a lista de estudos relacionados com os países que mais publicam sobre o tema de gamificação personalizada, nota-se que dentre os continentes que possuem mais países que estudam sobre esse tema é o continente europeu, e dentre todos os países a Alemanha tem mais estudos publicados nesta área, seguido como segundo lugar o Canadá, depois vem a Áustria e Bélgica. Brasil e França vêm empatados em terceiro lugar.

\section{Tabela 3. Apresenta os estudos primários que serviram como base para esse mapeamento sistemático}

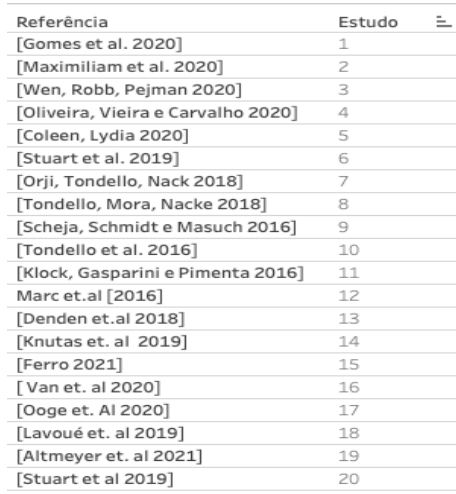

Fonte: Elaborada pelos autores com base nos estudos primários (2016 - 2021).

Tabela 4. Distribuição de estudos por país e ano de publicação

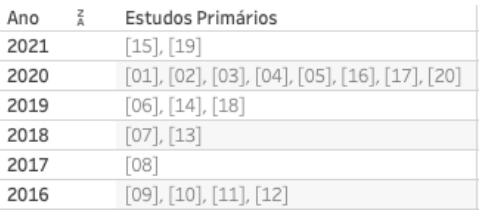

Fonte: Elaborada pelos autores com base nos estudos primários (2016 - 2021).

Tabela 5. Distribuição de estudos por país e ano de publicação

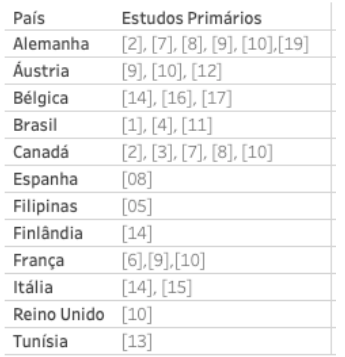

Fonte: Elaborada pelos autores com base nos estudos primários (2016 - 2021).

O Gráfico 1 apresenta uma correlação dos locais de publicação, estudos primários, ano e mecanismo de busca. Dentre os estudos primários, a maioria foi encontrada no congresso do CHI PLAY - Anais do Simpósio Anual de Interação Humano Computador no Jogo. Durante a criação do gráfico, foram agrupados os locais das publicações que tinham mais de um artigo nele durante o mesmo evento no ano.

Gráfico 1. Distribuição dos mecanismos de busca, ano e locais de publicação 


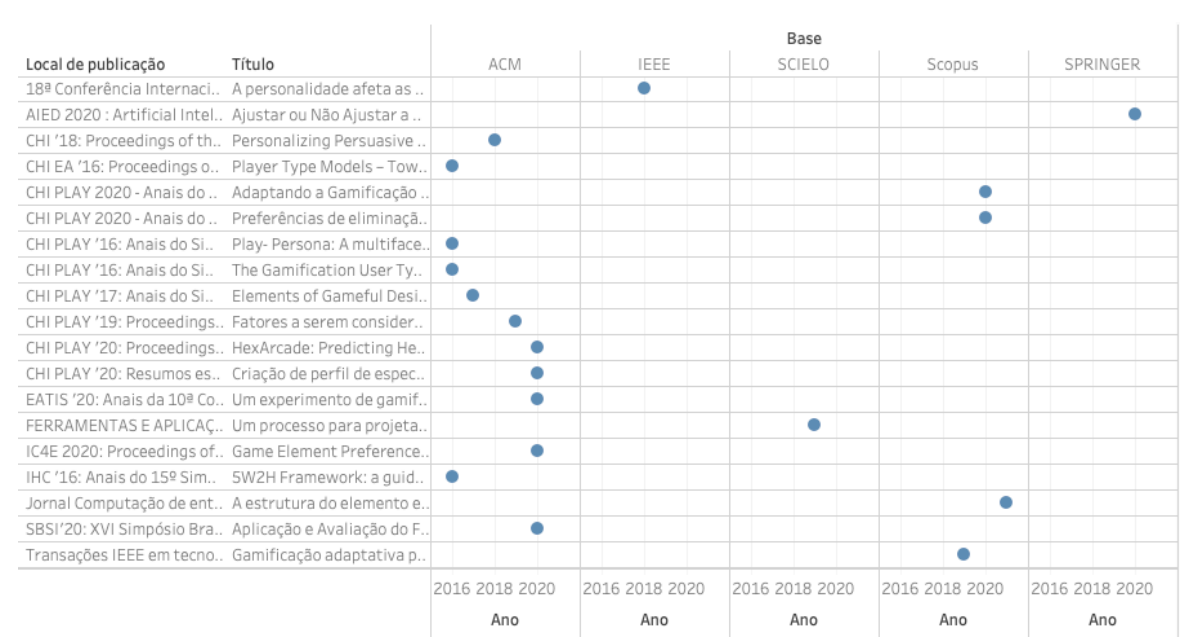

Fonte: Elaborada pelos autores com base nos estudos primários (2016 - 2021).

\subsection{Apreciação dos resultados}

Através dos resultados gerais obtivemos informações sobre qual foi a base que mais teve publicações com tema gamificação personalizada. Apenas em 2018 não foram encontrados estudos relacionados com o tema pesquisado. O CHI PLAY possui o total de $45 \%$ dos estudos primários relevantes para a pesquisa, os outros locais de publicação tiveram apenas um estudo em cada. Também identificamos os autores que mais escrevem sobre esse tema e a Tabela 6 mostra os estudos dos autores que mais publicaram. Verificaram-se outros padrões que são os seguintes: do ano de 2016 até 2019 houve uma produção menor que os anos de 2020 até 2021, mas sendo o ano de 2020 o mais produtivo com $40 \%$ do total dos estudos primários. Esse mapeamento só conseguiu obter publicações até março de 2021, mostrando um crescimento considerável no interesse de pesquisadores neste tema.

A maioria dos pesquisadores que lideram o ranking individualmente, acerca dos que mais produziram estudos sobre o tema durante 2016 até 2021 estão no Canadá. Uma correlação provável com este fato é que o Canadá é o segundo país onde mais encontramos estudos primários, mas lembrando que o continente europeu possui o maior número de pesquisadores que estudam sobre o tema e no somatório dos que mais produziram. A Tabela 6 mostra a lista dos autores e seus respectivos estudos que estão incluídos nesta pesquisa.

Tabela 6. Autores que mais publicaram sobre gamificação personalizada de 2016-2021.

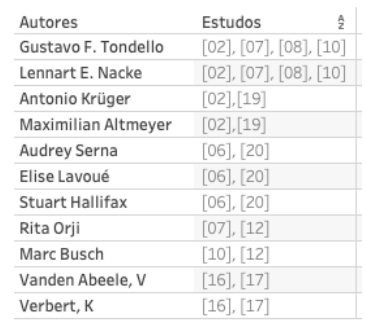

Fonte: Elaborada pelos autores com base nos estudos primários $(2016$ - 2021).

Na seção subsequente tratamos das questões de pesquisa abordadas neste mapeamento e como foram compreendidas suas respostas. 


\section{Questões de Pesquisa}

As sub-seções adiante apresentam os dados tradados que foram obtidos a partir dos estudos primários selecionados após aplicar CI e CE.

\subsection{QP1 - Quais foram os modelos ou frameworks de gamificação personalizada utilizados na gamificação?}

A QP1 tem o objetivo de encontrar os frameworks ou modelos que estão sendo utilizados para a gamificação personalizada, porém durante essa investigação verificamos outras informações interessantes como três novos modelos que foram os de e.g. Gomes et al. (2020), Klock, Gasparini e Pimenta (2016) e Ferro (2021). Descobrimos também que vários estudos primários utilizam de modelos ou teorias para verificar a satisfação do usuário durante o estudo, que são o modelo dos cinco grandes fatores (Big Five Factor Model - BFI) e a teoria da autodeterminação (Selfdetermination theory - SDT). Já os frameworks utilizados foram: os Hexad, BrainHex e Yee Motivation to Play Online Games Inventory (YMPOGI).

Durante a QP1 foi identificado que alguns estudos utilizaram mais de um framework em sua gamificação como visto em e.g. Scheja, Schmidt e Masuch (2016) e em Marc et al. (2016). Verificamos que sua maior incidência ocorreu no ano de 2020. Os frameworks encontrados durante a QP1 são descritos a seguir:

\section{YMPOGI}

Com base em e.g. Bartle (1996) o pesquisador e.g. Yee (2006) utilizou em seu trabalho o YMPOGI. Esse trabalho foi desenvolvido e aplicado com um questionário com 40 perguntas, que tinha como objetivo analisar o interesse dos jogadores com base no que foi proposto inicialmente por e.g. Bartle (1996). Segundo Yee (2006) identificou dez subcomponentes motivacionais após aplicar o questionário, posteriormente esses 10 subcomponentes foram compilados em três categorias que são: a realização (e dentro desta temos os dois subcomponentes de avanço e mecânica), a segunda é a social, (e dentro desta temos os quatro subcomponentes: competição, sociabilização, relacionamento e trabalho de equipe) e a última categoria que é a imersão (dentro desta, temos os quatro subcomponentes: de descoberta, role-playing, personalização, escapismo).

\section{BrainHex}

O framework proposto por e.g. Nacke, Bateman e Mandryk (2014) é conhecido como BrainHex e classifica os jogadores com base em sua personalidade e motivação no jogo. O BrainHex se fundamenta em resultados neurobiológicos e também por um modelo de jogador orientado por razões demográficas, e.g. [Nacke, Bateman e Mandryk 2014]. A pesquisa aplicou um questionário e após sua análise foram elencados sete tipos, que são: seeker, survivor, daredevil, mastermind, conqueror, socializer $e$ achiever. Existe um fator importante nessa pesquisa que é relacionar o entusiasmo do jogador e os fatores fisiológicos para assim sugerir o tipo que mais se aproxime da personalidade do jogador, e.g. [Nacke, Bateman e Mandryk 2014].

Hexad

Na pesquisa realizada por e.g. Marczewski (2015) foi realizado um estudo com foco na gamificação, algo diferente das pesquisas de e.g. Bartle (1996), Yee (2006) e 
Nacke, Bateman e Mandryk (2014). Esta pesquisa descreve a motivação do jogador no ambiente gamificado - esse modelo foi denominado de Hexad e tem um questionário que classifica o jogador com base na pontuação em seis perfis do framework que são: empreendedor, socializador, filantropia, espírito livre, disruptor e jogador. Esses perfis são motivados por recompensas extrínsecas nas quais a realização de atos e tarefas vêm através de algum tipo de barganha, e.g. Marczewski (2015).

Podemos encontrar esse questionário já traduzido para o português e testado por e.g. Tondello et al. (2019); a sua utilização vem sendo uma técnica de classificação em ambientes gamificados, conforme encontramos em e.g. Toda et al. (2018]).

\subsection{QP2: Dentre as pesquisas encontradas ocorreu algum tipo de adaptação do modelo ou framework utilizado?}

A QP2 verificou quais os frameworks encontrados tinham adaptações. Dentre os estudos encontrados, o framework de Yee foi encontrado apenas no estudo de e.g. Scheja, Schmidt e Masuch (2016). O framework de BrainHex foi encontrado em quatro estudos, mas nestes dois estudos de e.g. Scheja, Schmidt e Masuch (2016) e em Marc et al. (2016) verificou-se algum tipo de adaptação no framework. O framework Hexad que foi desenvolvido especificamente para a gamificação obteve diversos tipos de adaptações. O Gráfico 2 mostra os estudos que apresentaram algum tipo de alteração e seus respectivos frameworks relacionados.

Gráfico 2. Distribuição estudos e os frameworks que sofreram ou não adaptação e suas respectivas bases de dados mecanismos de busca, ano e locais de publicação

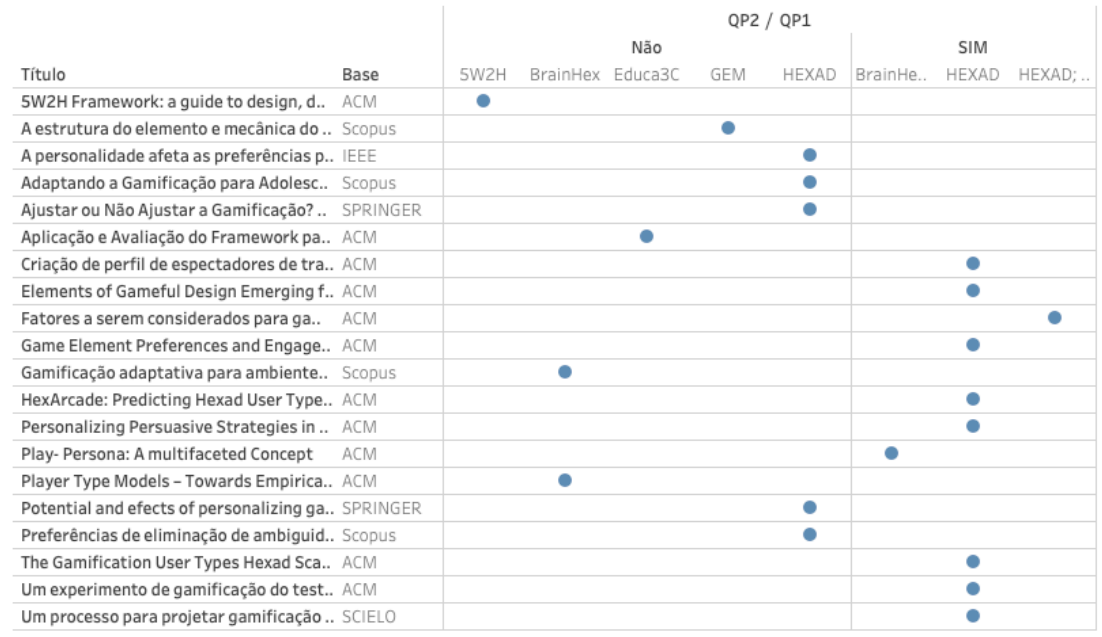

Fonte: Elaborada pelos autores com base nos estudos primários (2016 - 2021).

Durante a pesquisa foi identificada a utilização de teoria e modelo, como o modelo de cinco fatores grandes e a teoria da autodeterminação. Esses foram encontrados em 10 estudos primários durante esta pesquisa; na Tabela 7 apresentamos os estudos sendo 5 destes referentes ao BFI e os outros 5 do SDT.

Tabela 7. Apresentação dos estudos que utilizam o BFI e o SDT.

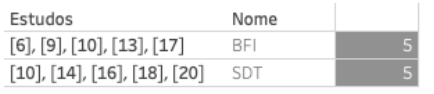


Fonte: Elaborada pelos autores com base nos estudos primários (2016 - 2021).

\subsection{QP3: Em quais setores foram utilizadas a gamificação personalizada?}

Em geral, a maioria dos estudos explorou setores da educação nos segmentos de educação do adolescente, cursos técnicos e cursos superiores. Em seguida vem o interesse em aplicativos gamificados adaptados com base no perfil do usuário. Consecutivamente vem validação com três estudos, dois estudos sobre propostas de framework, bem como, ferramentas e streaming com um estudo em cada setor. $\mathrm{O}$ Gráfico 3 apresenta os estudos primários relevantes para a pesquisa e seus respectivos setores, bem como intersecção entre as QP1, QP2 e QP3 abordadas neste mapeamento sistemático.

Gráfico 3. Distribuição estudos com relação as três questões de pesquisa abordadas no mapeamento.

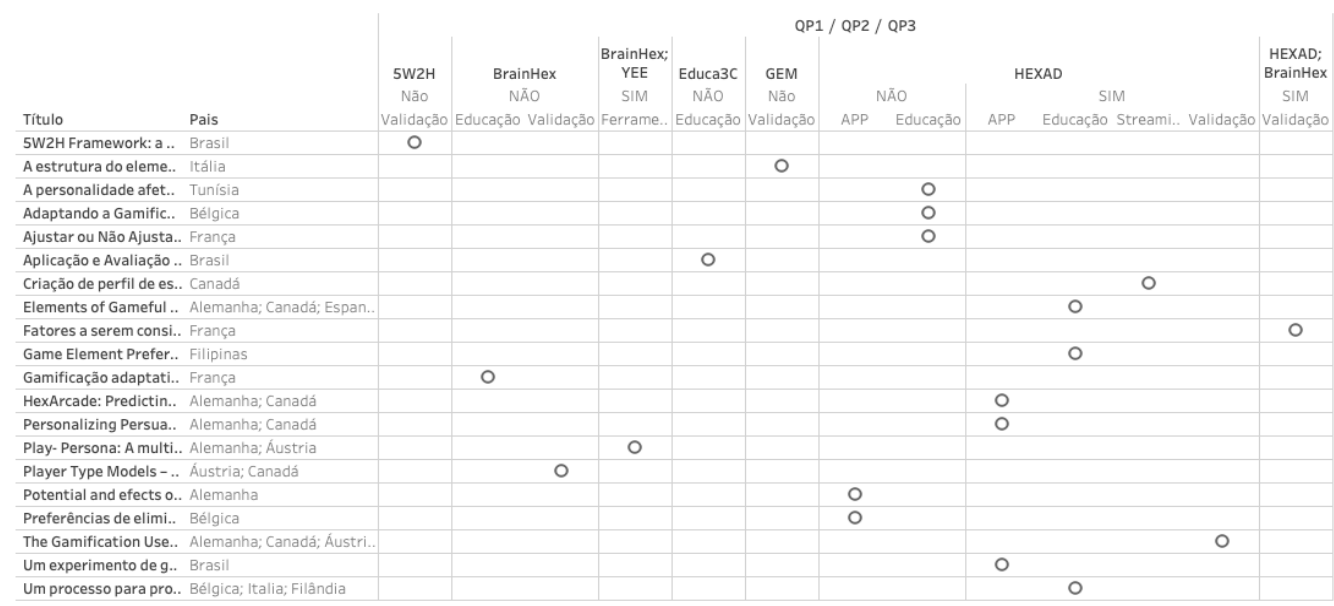

Fonte: Elaborada pelos autores com base nos estudos primários (2016 - 2021).

Neste gráfico 3 podemos identificar os estudos primários selecionados, bem como o país que realizou a pesquisa e a intersecção das questoes de pesquisas abordadas, quais os frameworks utilizados, se ocorreu algum tipo de adaptação e em quais setores.

\section{Conclusão}

Esse artigo apresentou um estudo de mapeamento sistemático para uma visão sobre uso de frameworks de personalização de gamificação. As principais conclusões são as seguintes: i) O Hexad é o framework mais utilizada quando se trata de gamificação personalizado; ii) a maioria das pesquisas que computam 52,38\% dos estudos fizeram uso de algum tipo de adaptação nos modelos de framework utilizados; iii) verificou-se que a maioria dos estudos focam no setor de educação e de aplicativos. O setor de educação tem um maior foco para as peculiaridades e variedade de alunos e usuários.

Em razão do caráter exclusivo da pesquisa, muitos artigos não foram incluídos. Porém os artigos que foram selecionados trouxeram dados importantes relacionados a gamificação personalizada e para aqueles que pretendem desenvolver pesquisas nesta área. 
Afinal, se faz necessário estar preparado para adaptar a gamificação motivando cada vez mais o usuário e a fim de propiciar que uma atividade mude de desagradável para agradável.

\section{Referências}

Andrade, T. S. D. (2019). Um mapeamento sistemático sobre o uso da gamificação na Plataforma Moodle.

Altmeyer, M; Gustavo F. Tondello; Antonio Krüger e Lennart E. Nacke. (2020). HexArcade: Predição de tipos de usuários hexadecimais usando aplicativos de jogo. In Proceedings of the Annual Symposium on Computer-Human Interaction in Play (CHI PLAY '20). p. 219-230. DOI: https: //doiorg.ez291.periodicos.capes.gov.br/10.1145/3410404.3414232, março.

Altmeyer, M., Lessel, P., Jantwal, S. et al. (2021). Potencial e efeitos da personalização de aplicativos de fitness para jogos usando intenções de mudança de comportamento e tipos de usuário hexadecimais. User Model User-Adap Inter. https://doi.org/10.1007/s11257-021-09288-6, março.

Bartle, R. (1996). Hearts, clubs, diamonds, spades: Players who suit muds. Journal of MUD research, 1(1):19.

Coleen M. Amado e Lydia S. Roleda. (2020). Preferências de elementos de jogo e envolvimento de diferentes tipos de jogadores hexadecimais em um curso de física gamificado. Em Proceedings of the 2020 11th International Conference on EEducation, E-Business, E-Management, and E-Learning ( IC4E 2020 ). p. 261-267. DOI: https: //doi-org.ez291.periodicos.capes.gov.br/10.1145/3377571.3377610, março.

Denden .M, Tlili. A, Essalmi. F and Jemni. M. (2018) "Does Personality Affect Students' Perceived Preferences for Game Elements in Gamified Learning Environments?," 2018 IEEE 18th International Conference on Advanced Learning Technologies (ICALT), pp. 111-115, doi: 10.1109/ICALT.2018.00033, março.

Ferro. Lauren S. (2021) The Game Element and Mechanic (GEM) framework: A structural approach for implementing game elements and mechanics into game experiences, Entertainment Computing. Volume 36,100375, ISSN 1875-9521. https://doi.org/10.1016/j.entcom.2020.100375, março.

Gomes, Fernanda Pereira; Brito, Parcilene Fernandes; Fagundes, Fabiano; Acco Tives, Heloise and Canedo, Edna Dias. (2020). Aplicação e Avaliação do Framework para Gamificação de Sistemas Educacionais usando Análise Comportamental. No XVI Simpósio Brasileiro de Sistemas de Informação (SBSI'20). Artigo 47, p. 1-8. DOI: https: //doi-org.ez291.periodicos.capes.gov.br/10.1145/3411564.3411658, março.

Hamari, J., Koivisto, J., \& Sarsa, H. (2014, January). Does gamification work? --a literature review of empirical studies on gamification. In 2014 47th Hawaii international conference on system sciences, p. 3025-3034. Ieee.

Kitchenham, B. (2007). "Guidelines for performing Systematic Literature Reviews in Software Engineering," V 2.3 EBSE Technical Report, EBSE-2007-01. 
Knutas, A., van Roy, R., Hynninen, T. et al. (2019). A process for designing algorithmbased personalized gamification. Multimed Tools Appl 78, p. 13593-13612. https://doi.org/10.1007/s11042-018-6913-5, março.

Klock, Ana Carolina Tomé; Gasparini, Isabela e Pimenta, Marcelo Soares. (2016). 5W2H Framework: um guia para projetar, desenvolver e avaliar a gamificação centrada no usuário. Em Anais do $15^{\circ}$ Simpósio Brasileiro de Fatores Humanos em Sistemas de Computação (IHC'16). Artigo 14, p. 1-10. DOI: https: //doiorg.ez291.periodicos.capes.gov.br/10.1145/3033701.3033715, março.

Lamprinou, D. and Paraskeva, F. (2015). Gamification design framework based on sdt for student motivation. p. 406-410.

Lavoué, E., Monterrat, B., Desmarais, M., George, S. (2019). Gamificação adaptativa para ambientes de aprendizagem. IEEE Transactions on Learning Technologies, 12 (1), art. 8334657, pp. 16-28. http://ieeexplore-ieeeorg.ez291.periodicos.capes.gov.br/xpl/RecentIssue.jsp?punumber=4620076 doi: 10.1109 / TLT.2018.2823710, março.

Marc Busch, Elke Mattheiss, Rita Orji, Peter Fröhlich, Michael Lankes e Manfred Tscheligi. (2016). Modelos de tipo de jogador: em direção à validação empírica. Em Proceedings of the 2016 CHI Conference Extended Abstracts on Human Factors in Computing Systems (CHI EA '16 ). Association for Computing Machinery, Nova York, NY, EUA, 1835-1841. DOI: https: //doiorg.ez291.periodicos.capes.gov.br/10.1145/2851581.2892399, março.

Marczewski, A. Even Ninja Monkeys Like to Play: Gamification, Game Thinking and Motivational Design. (2015). CreateSpace Independent Publishing Platform.

Mora, A., Riera, D., González, C., \& Arnedo-Moreno, J. (2017). Gamification: a systematic review of design frameworks. Journal of Computing in Higher Education, 29(3), p. 516-548.

Nacke, L. E., Bateman, C., and Mandryk, R. L. (2014). Brainhex: A neurobiological gamer typology survey. Entertainment computing, 5(1):55-62.

Oliveira, A. F. D. (2018). Gamificação no cotidiano escolar: Um mapeamento sistemático de literatura com ênfase em tecnologia e educação.

Oliveira, Luma Wanderley de; Vieira Marcos Alves e Sérgio Teixeira de Carvalho. (2020). Um experimento de gamificação do teste de tipologia de marczewski. In Proceedings of 10th Euro-American Conference on Telematics and Information Systems (EATIS '20). Art. 40, p. 1-5. DOI: https: //doiorg.ez291.periodicos.capes.gov.br/10.1145/3401895.3402072, março.

Ooge, J., De Croon, R., Verbert, K., Vanden Abeele, V. (2020). Adaptando Gamificação para Adolescentes: um Estudo de Validação de Big Five e Hexad em holandês. In Proceedings of the Annual Symposium on Computer-Human Interaction in Play (CHI PLAY '20). Association for Computing Machinery, Nova York, NY, EUA, $\quad$ p. 206-218. DOI: org.ez291.periodicos.capes.gov.br/10.1145/3410404.3414267, março.

Orji Rita; Tondello F. Gustavo e Nacke E. Lennart. (2018). Personalização de estratégias persuasivas em sistemas de jogo para tipos de usuário de gamificação. 
Proceedings of the 2018 CHI Conference on Human Factors in Computing Systems. Art. 435, p. 1-14. DOI: https: //doiorg.ez291.periodicos.capes.gov.br/10.1145/3173574.3174009, março.

Petersen, S. Vakkalanka, and L. Kuzniarz. (2015). Guidelines for conducting systematic mapping studies in software engineering: An update. Information and Software Technology, 64: p. 1-18.

Poffo, Marcio; Agostini Volani, Elisângela. (2018). Gamificação para motivar o aprendizado. CIET: EnPED, São Carlos, maio. ISSN 2316-8722. Disponível em: $<$ https://cietenped.ufscar.br/submissao/index.php/2018/article/view/268>. Acesso em: 02 jul. 2021.

Scheja Stephanie; Schmidt Ralf e Masuch Maic. (2016). Play-persona: um conceito multifacetado. Em Anais da 20 a Conferência Internacional Academic Mindtrek (Academic Mindtrek '16). p. 375-384. DOI: https: //doiorg.ez291.periodicos.capes.gov.br/10.1145/2994310.2994356, março.

Seaborn, K., \& Fels, D. I. (2015). Gamification in theory and action: A survey. International Journal of human-computer studies, 74, p. 14-31.

Souza, M., Constantino, K., Veado, L., and Figueiredo, E. (2017). Gamification in software engineering education: An empirical study.

Stuart Hallifax, Audrey Serna, Jean-Charles Marty, Guillaume Lavoué e Elise Lavoué. (2019). Fatores a serem considerados para gamificação sob medida. In Proceedings of the Annual Symposium on Computer-Human Interaction in Play (CHI PLAY '19). Association for Computing Machinery, Nova York, NY, EUA, 559-572. DOI: https: //doi-org.ez291.periodicos.capes.gov.br/10.1145/3311350.3347167, março.

Toda, A. M., Valle, P. H. D. D. and Isotani, S. (2017). The Dark Side of Gamification: An Overview of Negative Effects of Gamification in Education. In Communications in Computer andInformation Science. Springer, p.143-156.

Toda, A. M., Do Carmo, R. M. C., Da Silva, A. P., Bittencourt, I. I. and Isotani, S. (2018). An approach for planning and deploying gamification concepts with social networks within educational contexts. International Journal of Information Management.

Tondello, Gustavo F; Wehbe, Rina R; Diamond, Lisa; Busch, Marc; Marczewski, Andrzej e Nacke, Lennart E. (2016). A escala hexadecimal de tipos de usuário de gamificação. Nos anais do Simpósio Anual de 2016 sobre Interação HumanoComputador no Jogo (CHI PLAY '16). p. 229-243. DOI: https: //doiorg.ez291.periodicos.capes.gov.br/10.1145/2967934.2968082

Tondello F. Gustavo; Mora Alberto e Nacke E. Lennart. (2017). Elementos do Gameful Design surgindo das preferências do usuário. Proceedings of the Annual Symposium on Computer-Human Interaction in Play. p. 129-142. DOI: https: //doiorg.ez291.periodicos.capes.gov.br/10.1145/3116595.3116627

Tondello, G. F., Mora, A., Marczewski, A., and Nacke, L. E. (2019). Empirical validation of the gamification user types hexad scale in english and spanish. International Journal of Human-Computer Studies, 127:95-111. 
Van Houdt, L., Millecamp, M., Verbert, K., \& Vanden Abeele, V. (2020). Disambiguating preferences for gamification strategies to motivate proenvironmental behaviour. Paper presented at the CHI PLAY 2020 - Proceedings of the Annual Symposium on Computer-Human Interaction in Play, 241-253. doi:10.1145/3410404.3414244, março.

Wen Bo Yu; James Robb e Pejman Mirza-Babaei. (2020). Profiling Livestream Spectators. Em Extended Abstracts do 2020 Annual Symposium on ComputerHuman Interaction in Play (CHI PLAY 20). p. 403-407. DOI: https: //doiorg.ez291.periodicos.capes.gov.br/10.1145/3383668.3419904, março.

YEE, N. Motivations for play in online games. (2006). [S.1.]: Cyberpsychology and Behavior. 